\title{
DERIVADOS DE LA YUCA Y COMPONENTES TÓXICOS EN BRASIL
}

\section{CASSAVA DERIVATIVES AND TOXIC COMPONENTS IN BRAZIL}

\author{
João Tomaz da Silva' ${ }^{1}$ Cláudia Denise de Paula², Talita Moreira de Oliveira ${ }^{1}$ y Omar Andrés Pérez ${ }^{2}$ \\ Recibido para evaluación: Julio 17 de 2008 - Aceptado para publicación: Diciembre 8 de 2008
}

\section{RESUMEN}

El presente trabajo es una revisión sobre componentes tóxicos de la yuca y medidas adoptadas en el procesamiento para reducción de éstos a concentraciones seguras para el consumidor. La yuca (Manihot esculenta Crantz) se constituye en alimento básico de millones de personas en todo el mundo, principalmente en países en vía de desarrollo. Una de las características de la planta es la presencia del glucósido Linamarina, con alto poder de hidrólisis liberando cianuro $\left(\mathrm{CN}^{-}\right)$, lo que permite clasificar en los diferentes cultivos en grupos mansa (concentraciones menores que $100 \mathrm{mg} \mathrm{HCN} \mathrm{Kg}^{-1}$ ) y brava (concentraciones mayores que $100 \mathrm{mg} \mathrm{HCN} \mathrm{Kg}^{-1}$ ). Aunque este glucósido sea removido en su mayor parte durante el procesamiento de raíces, debido al uso de condiciones inadecuadas de proceso, sobretodo de variedades bravas; el puede estar presente, en formas intacta o derivada $\left(\mathrm{CN}^{-}\right)$, en productos derivados de la planta en concentraciones impropias para el consumo, poniendo en riesgo la salud del consumidor. En Brasil, la yuca es materia prima de innúmeros derivados: harinas de mesa, fécula, polvillos, beiju, tapioca, carimã o masa puba, hojas o harinas de hojas y tucupi. Una análisis de los flujogramas generales de proceso de tucupi, harina, polvillos y fécula permite concluir que etapas como fermentación y calentamiento a ebullición (en el caso del tucupi), desmembramiento, prensado y torrefacción (en caso de harinas), triturado, lavado, fermentación y secado (en caso de polvillo y fécula), son efectivas en la reducción o eliminación total del potencial toxicológico de variedades con altas concentraciones de cianógenos.

Palabras clave: yuca, linamarina, ácido cianídrico, toxidez.

\footnotetext{
${ }^{1}$ Departamento de Tecnología de Alimentos, Universidad Federal de Viçosa, avenida Peter Henry Rolfs, Campus Universitario 36570-000, Viçosa, MG, Email: jtsborges@yahoo.com.br

${ }^{2}$ Departamento de Ingeniería de Alimentos, Universidad de Córdoba Km 12 vía a Ciénaga de Oro, Tel (4) 894 0508, Fax (4) 786 0255. Email: operez@sinu.unicordoba.edu.co
} 


\begin{abstract}
This work was aimed at presenting a brief review on toxic components of cassava and steps taken in processing for reducing these concentrations to a safe (ave) for consumers. The cassava (Manihot sculenta) is an staple food for millions of people worldwide, mostly in developing countries. One of the characteristics of the plant is the presence of Linamarina glycoside, with high-powered hydrolysis releasing cyanide $\left(\mathrm{CN}^{-}\right)$, allowing classified into different cultivar groups mansa (concentrations lower than 100 $\mathrm{mg} \mathrm{HCN} \mathrm{Kg}{ }^{-1}$ ) and brava (concentrations above $100 \mathrm{mg} \mathrm{HCN} \mathrm{Kg}^{-1}$ ). Although this glycoside is mostly removed during root processing, due to the use of inadequate conditions of process, especially bravas varieties may be present in products derived from the plant at unfit concentrations for consumption, putting at risk consumers health. In Brazil, cassava is raw material for countless derivatives: meal table, starch, powders, beiju, tapioca, or mass carimã Pub, leaves and tucupi meal. An analysis of the overall process flowcharts for tucupi, flour, powders and starch leads to the conclusion that fermentation and boiling (in the case of tucupi), dismemberment, roasting and pressing (for flour), crushed, washed, fermentation and drying (dust and starch), are effective in reducing or removing toxicological concentrations of cyanide.
\end{abstract}

Key words: cassava, linamarina, hydrogen cyanide, toxidez.

\title{
INTRODUCCIÓN
}

La yuca (Manihot esculenta Crantz), perteneciente a la familia Euphorbiaceae, es un importante cultivo tropical constituyéndose en alimento básico de millones de habitantes en todo el mundo y siendo cultivada en más de 90 países tropicales y subtropicales. Brasil se destaca como el segundo mayor productor con cerca de $13 \%$ de la producción mundial, siendo esta tuberosa utilizada principalmente sobre la forma de harina y otros productos industrializados. En algunas regiones del nordeste brasilero es uno de los principales cultivos, de la cual depende la subsistencia y renta de productores y la alimentación animal (Cabral et al., 2002; Lorenzi, 2003).

Además de la riqueza en carbohidratos que poseen las raíces, particularmente almidón, la parte aérea es fuente de proteínas, vitaminas y minerales, destacándose como una nueva perspectiva para alimentación humana y animal, y como materia prima para muchos productos industriales y generación de empleo y renta (Ferreira, 1997; EMBRAPA, 2006). Según el Instituto Brasilero de Geografía y Estadística (IBGE, 2006), la producción nacional de este cultivo fue superior a 23 millones ton con un rendimiento medio de 13.6 ton de raíces ha-1 en 2004. Los estados y ciudades de las regiones norte y nordeste se destacan como los principales productores de yuca en Brasil (Figura 1). El cultivo de ésta en zonas de sequía prolongada como el Nordeste de Brasil y algunos países de África, Sudeste Asiático y América Latina, es favorecido por su capacidad en usar agua eficientemente y la facilidad de adaptación a suelos de baja fertilidad (Cardoso, 2003; Barros, 2004).

La raíz típica de yuca es larga, con diámetro más o menos uniforme en la región mediana, la región proximal (que se liga al restante de la planta) presenta diámetro mayor que la intermediaria y distal (parte final) (Figura 2a). La sección transversal de la raíz (Figura 2b) 


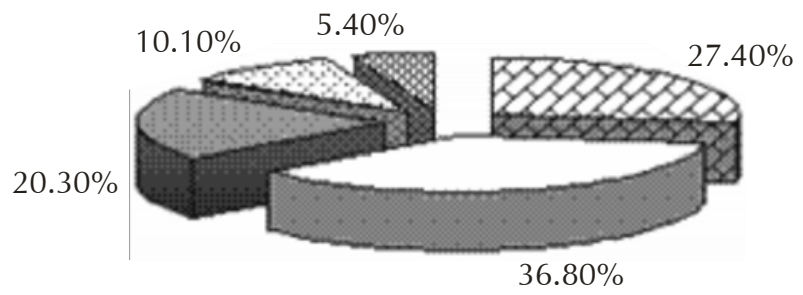

$\square$ Norte $\square$ Nordeste $\square$ Sur $\square$ Sudeste $\square$ Centro Oeste

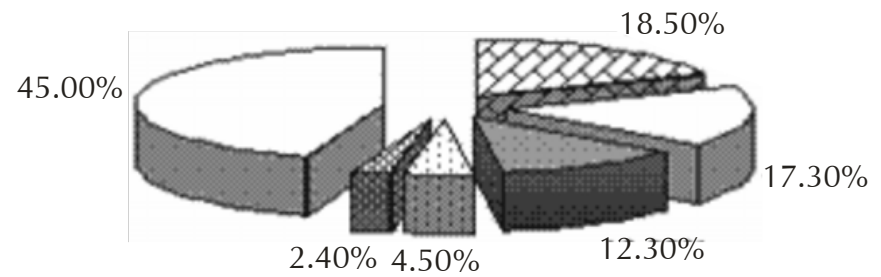

- Pará $\square$ Bahía $\square$ Paraná Sao Paulo $\square$ Mato Grosso $\square$ Otros

Figura 1. Regiones y principales Estados productores de yuca en el año 2004 (IBGE, 2006).
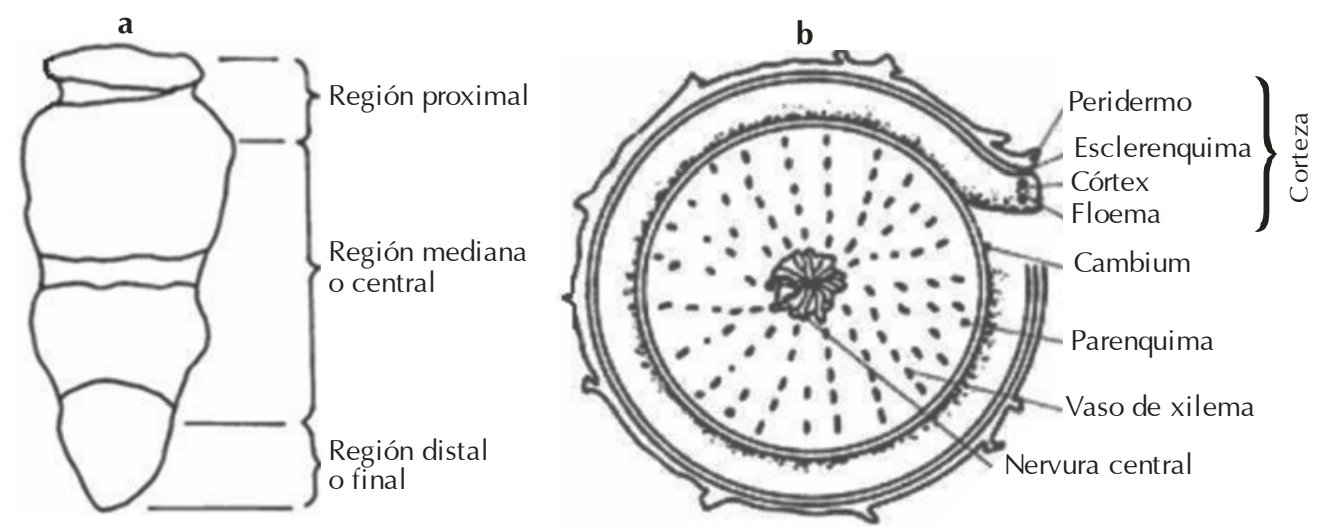

Figura 2. Vista parcial (a) y corte transversal (b) de raíz de yuca (CIAT, 2008).

revela la presencia de una nervadura central lignificada envuelta por el parénquima, principal región de almacenamiento de almidón de esta tuberosa, con coloración variando de blanca a rosa, pasando por colores crema, amarilla y naranja. La capa posterior comprende el córtex o entre capa, un conjunto de capas de células parenquimáticas sobrepuestas, con superficie variando de rosado, levemente marrón o blanca (Dallaqua y Coral, 2002; Lorenzi, 2003). Por último se tiene la epidermis, que puede ser lisa o áspera, generalmente de color marrón oscuro. Con el aumento de largo y diámetro, la raíz puede presentar pequeñas fisuras longitudinales sobre las cuales son sobrepuestas nuevas capas celulares de córtex, manteniendo la integridad de la raíz. 
Las variedades de yuca son normalmente clasificadas por el contenido de cianuro $\left(\mathrm{CN}^{-}\right)$ en la pulpa de la raíz, en mansas (aipim, macaxeira, dulces, inocuas o de mesa), intermedia y bravas (industriales). La mayor parte de la yuca de mesa es comercializada en fresco, siendo consumida después del uso de técnicas de preparado más simples (pelado, cocimiento, asado, fritura, etc.). Por otro lado, variedades industriales son utilizadas en la obtención de harinas, fécula o polvillos, siendo solamente consumidas después de algún tipo de procesamiento industrial detoxificante (Lorenzi, 2003; Valle et al., 2004; Fukuda y Otsubo, 2006). En Brasil, los principales derivados de las raíces de yuca son harinas de mesa (seca, de agua o mixta), fécula (polvillo dulce), polvillo acido, beiju, tapioca, carimã o masa puba, hojas o harinas de hojas y tucupi (Mattos et al., 2002). La harina tiene uso esencialmente alimentario con elevada especificidad regional que en muchos casos convierte al producto específico a mercados locales (Cardoso y Souza, 2002).

Las técnicas de procesamiento industrial para disminución del principio tóxico se basan en la disolución en agua o en la volatilización envolviendo procesos como la maceración, remojo en agua, calentamiento a ebullición, asado o fermentación de las raíces, o aún la combinación de estos procesos. A pesar de su elevada utilización como alimento, el consumo de yuca, sobre todo con elevado nivel de cianógenos, está relacionado con desordenes patológicos en

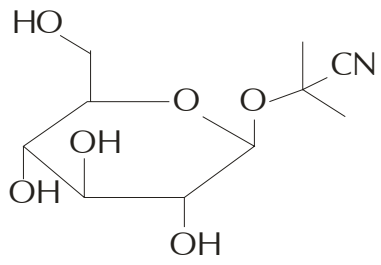

Linamarina (a) humanos y animales. Por tratarse de materia prima altamente perecedera (nivel de humedad en torno de 60\%), las raíces de yuca exigen procesamiento elaborado antes del consumo, mejorando de esta forma el almacenamiento, conveniencia en la manipulación, palatabilidad y seguridad nutricional (Iwuoha et al., 1997). La $\mathrm{DL}_{50}$ (dosis letal) aceptada por la OMS es de 10 mg HCN Kg-1 de peso vivo. Para fines industriales, el mayor o menor nivel de $\mathrm{HCN}$ no tiene importancia pues en el procesamiento industrial promueve su volatilización (Oliveira, 1986). Aparentemente, la mayor parte de HCN normalmente es liberada en ese procesamiento, pero es común encontrar algunos residuos que pueden ser suficientes para producir síntomas de intoxicación. El efecto tóxico del HCN puede ser agudo o crónico, caracterizándose el primero por envenenamiento drástico y el segundo por disturbios causados en el sistema nervioso central o por la ocurrencia de bocio (Cereda, 2003a). El presente trabajo tiene por objetivo presentar una revisión sobre los principales derivados de la yuca y la influencia del procesamiento en la reducción o eliminación total de sus componentes tóxicos a concentraciones seguras para su consumo.

\section{Compuestos tóxicos de la yuca}

La yuca acumula dos glucósidos cianogénicos en las raíces y hojas, la linamarina y la lotaustralina (proporción de 93:7) (Figura 3). Esos dos glucósidos son capaces de generar ácido cianídrico $(\mathrm{HCN})$



Lotaustralina $(b)$

Figura 3. Fórmulas estruturales de la linamarina (a) y lotaustralina (b). 
desde que ocurra una hidrólisis, de forma que la concentración de cianuro y el uso de la yuca como alimento están condicionados a una hidrólisis enzimática de esos dos glucósidos cianogénicos. La enzima responsable por la hidrólisis es la linamarasa y cuando el tejido es dilacerado, la linamarina entra en contacto con la enzima, la cual es separada del glucósido en el tejido intacto por ser localizada en lugar distinto de la célula. La ruptura produce glucosa y $\alpha$-hidroxinitrilo; esta última, cuando es catalizada por una hidroxinitrilo liasa se transforma espontáneamente en $\mathrm{HCN}$ y en las cetonas correspondientes, el proceso es llamado cianogénesis. La linamarina se encuentra presente en altas concentraciones en las hojas jóvenes, tallo y en pequeñas cantidades en la pulpa (Dias et al., 1997a; Cagnon et al., 2002; Taiz y Zeiger, 2004). De acuerdo con Carvalho (1992), la concentración de $\mathrm{HCN}$ en el parénquima puede variar de 14 a $400 \mathrm{mg}$ en peso fresco. Basado en el riesgo a la salud causado por la exposición al cianuro, y teniendo en cuenta la mayor seguridad para consumo, variedades de yuca que presentan hasta 100 mg HCN Kg-1 de pulpa son consideradas mansas; de 100 a 200 mg intermediaria y arriba de 200 mg bravas (Lorenzi, 2003). Una segunda clasificación es presentada por Cereda (2003b) y Valle et al. (2004) considera la existencia de apenas dos grupos: dulces o mansas (<100 mg HCN Kg-1) y bravas o amargas (>100 $\mathrm{mg} \mathrm{HCN} \mathrm{Kg}^{-1}$ ). Muchas investigaciones y esfuerzos han sido hechos para desarrollar plantas de yuca sin $\mathrm{CN}^{-}$, lo que aún no es posible (Du et al., 1995). De esta forma, además de la selección de variedades con baja concentración de $\mathrm{CN}^{-}$, es importante la aplicación de procedimientos adecuados durante el procesamiento que impidan la ocurrencia de intoxicaciones.

La yuca es probablemente la más conocida de las plantas cianogénicas, en razón de su amplia distribución en países tropicales. En África, varios problemas de salud están relacionados con el consumo de yuca y derivados debido a una coyuntura especial de subdesarrollo, falta de recursos para salud y alimentación, baja escolaridad y falta de informaciones en general. Por otro lado, en los países de América Latina, donde hay también gran consumo de esta raíz en la alimentación humana y animal, la situación es muy diferente. En el Brasil donde ocurren cinturones de desnutrición, los casos comprobados de intoxicación por consumo de yuca y derivados son raros, y su comprobación discutible (Cereda, 2003a).

\section{Diferencias fenotipicas entre variedades}

Normalmente no es posible distinguir entre variedades mansas y bravas por el aspecto externo de la planta, porque la concentración de $\mathrm{HCN}$ no se encuentra correlacionada con características morfológicas. En la práctica la separación entre ellas es hecha utilizando como criterio la toxicidad y la palatabilidad de las raíces. Este es un método subjetivo y la correlación no es exacta, tornando esa identificación de uso muy limitado (Lorenzi, 2003; Valle et al., 2004). De manera bastante subjetiva, variedades bravas tienen sabor amargo, mientras que las mansas son levemente dulces (Valle et al., 2004). El sabor amargo está asociado al potencial cianogénico pudiendo ser perceptible a partir de $100 \mathrm{mg}$ $\mathrm{HCN} \mathrm{Kg}^{-1}$ de pulpa de raíces (Dufour, 1988; Lorenzi et al., 1993).

El gran número de variedades de yuca existentes en Brasil permite la escogencia de aquellas que mejor se adaptan a la región y la finalidad de explotación de la agricultura. Variedades mansas, además de alta productividad y resistencia a plagas y enfermedades, deben presentar baja concentración de $\mathrm{HCN}$ en raíces y características cualitativas como bajo tiempo 
de cocimiento (10 a 30 minutos), cualidad culinaria de masa cocida y mayor resistencia al deterioro post-cosecha (Pereira et al., 1985; Normanha, 1988). Otras características tales como palatabilidad, plasticidad, pegajosidad, ausencia de fibras en la masa cocida, facilidad de pelado, raíces cortas y bien formadas son también importantes para el mercado consumidor, debiendo ser consideradas en la escogencia del cultivo (Mattos y Becerra, 2006). El ciclo vegetativo es otro factor determinante en el cultivo de yuca. Variedades industriales son cosechadas más tardíamente, generalmente presentando ciclo vegetativo de 18 a 24 meses (Souza y Fialho, 2006). Por otro lado, cultivos de mesa se caracterizan por el hecho de ser utilizadas para el consumo en fresco, siendo cosechadas con ciclo vegetativo de 7 a 14 meses (Barros, 2004).

Para la industria los cultivares de yuca deben ser seleccionados de acuerdo con la finalidad de utilización, pudiendo ser variedades mansas o bravas de alta productividad. Para la producción de harina y almidón, es importante que los cultivares presenten raíces con pulpa, córtex y película de coloración blanca, ausencia de cintas, película fina y raíces gruesas y bien formadas (Mattos y Becerra, 2006). A pesar de adaptarse a los más diferentes ecosistemas, la yuca presenta alta interacción del genotipo con el ambiente, con adaptación específica a determinadas regiones. Difícilmente un mismo cultivar se comporta de forma semejante en todos los ecosistemas. Este comportamiento puede estar relacionado al gran número de plagas y enfermedades restrictas a determinados ambientes, justificando en parte la gran diversidad de cultivares utilizada por los agricultores brasileros (Fukuda y Otsubo, 2006).

\section{REDUCCIÓN DE COMPUESTOS TÓXICOS DE LA YUCA}

El procesamiento adecuado es suficiente para reducir las concentraciones aceptables de cantidades de cianógenos tóxicos, existiendo etapas básicas para el procesamiento de harina de yuca, polvillo, fécula y tucupi (Figura 4). La OMS fijó la concentración segura de cianógenos en harina de yuca a $10 \mathrm{mg}$ (FAO/WHO, 1991), siendo que este valor puede variar según la legislación de cada país, como puede ser comprobado en Indonesia con límite aceptable de 40 mg (Damardjati et al., 1993; Djazuli y Bradbury, 1999).

El tucupi es una salsa de color amarilla extraída de la raíz de yuca brava, después que la yuca es pelada, triturada y prensada. Luego de la extracción, la salsa es decantada por un período de uno o dos días para remoción del almidón, desencadenando de esta forma un proceso de fermentación; siendo en seguida hervido y condimentado (Cagnon et al., 2002). Chisté et al. (2007) analizaron muestras comerciales de tucupi en la ciudad de Belém-PA, constatando concentraciones de cianuro total superior a $100 \mathrm{mg} \mathrm{HCN} \mathrm{Kg}^{-1}$ en $60 \%$ de las muestras. Entretanto, para los autores, este producto no ofrece riesgo a la salud del consumidor, una vez que es sometido a calentamiento a ebullición antes de ser ingerido. Una explicación para tales resultados puede estar relacionada al $\mathrm{pH}$ bajo del producto (alrededor de 3.6), pues según relatan Cagnon et al. (2002), la combinación entre este $\mathrm{pH}$ bajo y la alta concentración de linamarina puede llevar a la fijación del cianuro. El empleo de altas temperaturas, aunque inactive la acción de la linamarasa, promueve una hidrólisis más acentuada de los compuestos ciánicos y consecuente volatilización del cianuro libre. Los resultados encontrados por Chisté et al. (2007) revelaron una no estandarización en 


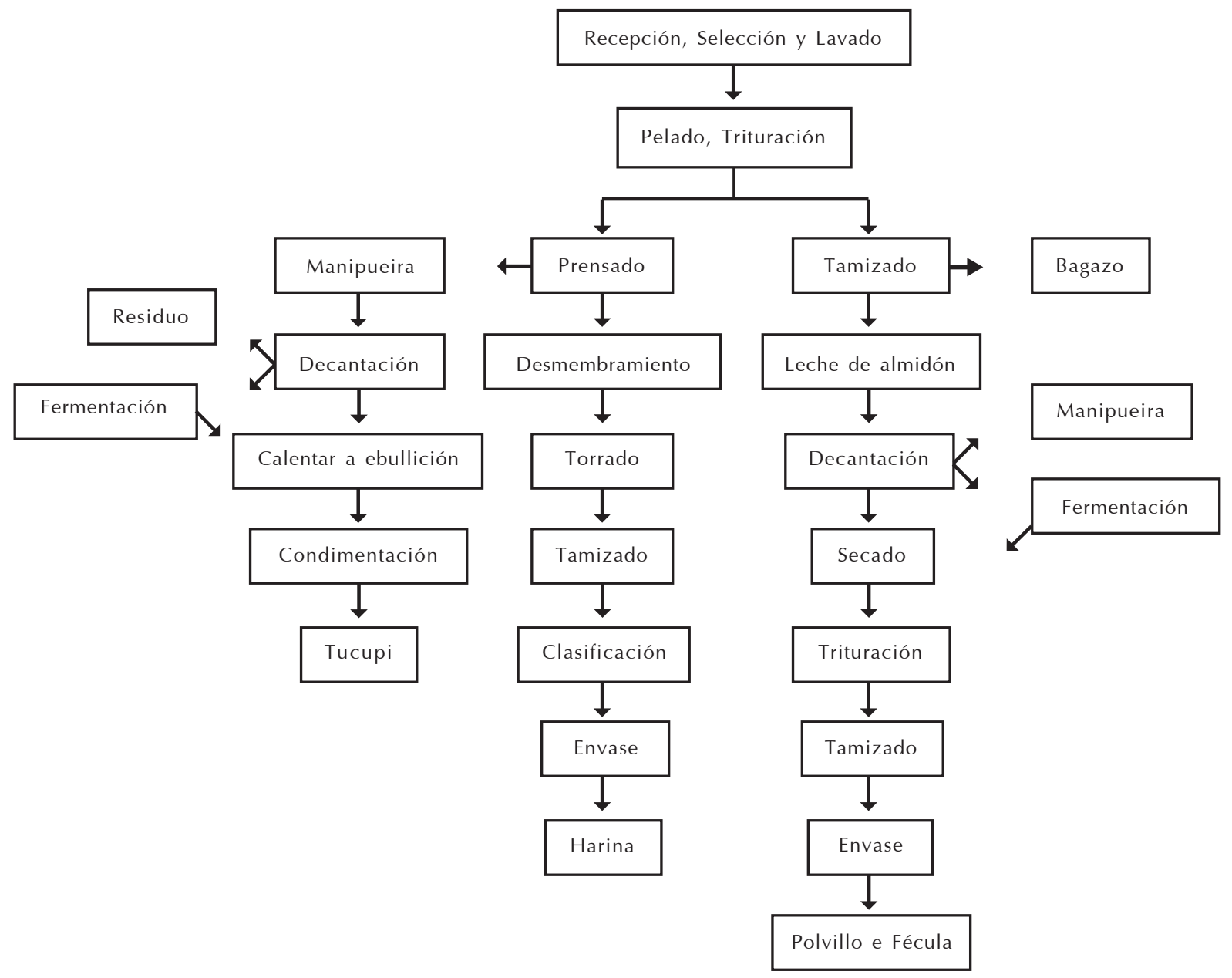

Figura 4. Flujograma básico de procesamiento de harina, polvillo y tucupi.

las características físico-químicas del tucupi comercializado en aquella región, tornándose necesarias mejorías en el proceso de fabricación del producto.

La harina de yuca es el producto obtenido por un ligero tostado de la ralladura de raíces de yuca previamente pelada, lavada y exenta del radical cianuro (Brasil, 1978). Con relación a eliminación del $\mathrm{CN}^{-}$, el proceso es efectivo porque al rallarla se colocan en contacto enzima y substrato en las mejores condiciones, $\mathrm{pH}$ entre 5.5 y 6.0 ( $\mathrm{pH}$ natural de la raíz) y temperatura ambiente. Correa (2000) estudió la actividad de la linamarasa en diferentes condiciones de $\mathrm{pH}$ y constató que el $\mathrm{pH}$ óptimo de la enzima fue 6.0; este valor se encuentra de acuerdo con aquellos registrados por Yeoh (1989) y Mkpong et al. (1990). Un análisis del flujograma presentado en la figura 4 permite concluir que además de las etapas de triturado y tostado, el prensado contribuye efectivamente para la reducción de la toxicidad, pues los glicocianetos solubles en agua son arrastrados con el agua de constitución de las raíces.

Cohen et al. (2007) encontraron concentraciones de $\mathrm{HCN}$ en las harinas de yuca del grupo seca y del grupo de agua variando de $7.75 \mathrm{mg} \mathrm{Kg}^{-1}$ a $20.60 \mathrm{mg} \mathrm{Kg}^{-1}$ y 3.45 a $12.17 \mathrm{mg}$, respectivamente. Conforme afirmaron los autores, estos valores se 
encuentran relacionados con factores como variedad, época de cosecha y condiciones de procesamiento de las raíces. Chisté et al. (2005) constataron un aumento en las concentraciones de $\mathrm{HCN}$ de $154.40 \mathrm{mg} \mathrm{Kg}^{-1}$ en la raíz en forma natural, para 167.68 mg $\mathrm{Kg}^{-1}$ en la raíz pelada, en función del aumento de la actividad cianogénica. En la masa prensada el valor fue reducido para $66.59 \mathrm{mg} \mathrm{Kg}^{-1}$, pues el contenido de linamarina y el HCN formado anteriormente, fueron, en su gran mayoría, arrastrados juntamente con el manipueira (líquido extraído de la masa triturada durante el proceso de prensado) que es destinada al preparo del tucupi; por lo tanto, el manipueira es un compuesto rico en $\mathrm{HCN}$. Una análisis de la harina obtenida revelo concentración de 5.19 (mg HCN Kg-1), presentando una dosificación baja de cianuro total por el hecho de haber sido tostada a altas temperaturas, volatilizando gran parte del HCN presente en la masa que había sido prensada anteriormente. Aproximadamente $70 \%$ de la linamarina presente en la raíz de yuca es removida por hidrólisis enzimática durante el procesamiento. En complementación, casi todo el $\mathrm{CN}^{-}$ generado es removido por volatilización o solubilización (Ikediob et al., 1980).

Según la Resolución 12/78 de la Comisión Nacional de Normas y estandarizaciones para Alimentos del Ministerio da Salud (CNNPA/MS, 1978), polvillo o fécula de yuca es el producto extraído de la yuca. Por medio de normas técnicas especiales relativas a alimentos y bebidas, el polvillo es clasificado en dulce y ácido, teniendo por base sólo la concentración de acidez. Para el producto fermentado, la acidez debe ser,

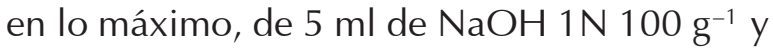
para el no fermentado de $1 \mathrm{ml}$ de $\mathrm{NaOH} 1 \mathrm{~N}$ $100 \mathrm{~g}^{-1}$. Para otras características, Ios valores son idénticos: humedad máxima de $14 \% \mathrm{p} / \mathrm{p}$, concentración mínima de almidón de $80 \% \mathrm{p} / \mathrm{p}$ y residuo mineral fijo máximo de $0.5 \% \mathrm{p} / \mathrm{p}$ (ABIA, 2000). La fermentación del almidón se hace en presencia de agua, dejando una capa de sobrenadante de 15 a $20 \mathrm{~cm}$ aproximadamente, formándose espuma en la superficie y desprendiéndose burbujas de gas del interior de la masa. Después de este tiempo de predominante fermentación láctica, el polvillo ácido es secado al sol, por un período que varía de acuerdo con el clima (Cereda y Lima, 1981, 1983; 1987; Demiate et al., 1999). Por otro lado, la fécula obtenida es secada en secadores de túnel o flash - dryer, (Leonel y Cereda, 2000; Balagopalan, 2002), donde los tipos de equipamientos utilizados en su extracción varían de acuerdo con la escala industrial. En ambos procesos es eficaz la reducción o eliminación de los componentes tóxicos a concentraciones seguras por la alta solubilidad peculiar, haciendo con que la manipueira contenga grandes cantidades de estas sustancias (Tylleskar et al., 1992; Bokanga, 1994). El secado correcto es otro factor importante en el procesamiento por favorecer la volatilización del HCN (Djazuli y Bradbury, 1999; Ngudi et al., 2003). AgborEgbe y Lape Mbome (2006) investigaron el efecto del procesamiento en la reducción de concentraciones de cianógenos en derivados de yuca, obteniendo una reducción del contenido cianogénico total superior al 97\%, reforzando la importancia de la desintegración de la raíz y del pH en la reducción o eliminación de la toxicidad; resultados semejantes fueron constatados por Jones et al. (1994). Todos los productos obtenidos a partir de yuca en Brasil, como harinas, carimã, puba, almidón y otros son seguros con relación a toxicidad por $\mathrm{CN}^{-}$, no ofreciendo riesgos a la salud del consumidor (Cereda, 1994b; Lorenzi, 2003). 


\section{CONCLUSIONES}

- La yuca (Manihot esculenta Crantz) posee elevada concentración de almidón en las raíces siendo considerado un alimento energético para millones de personas en el mundo, sobre todo en los países en vía de desarrollo. Presenta una serie de ventajas en relación a otros cultivos: fácil adaptación y propagación, rendimientos satisfactorios aún en suelos de baja fertilidad, potencial de resistencia o tolerancia a plagas y enfermedades, siendo cultivada en todos los estados brasileros.

- Una de las características de la planta de yuca es la presencia del glucósido Linamarina, potencialmente hidrolizable liberando cianuro $\left(\mathrm{CN}^{-}\right)$, lo que permite la clasificación de yuca en grupos mansa (<100 mg HCN Kg-1) y brava ( $>100 \mathrm{mg}$ $\left.\mathrm{HCN} \mathrm{Kg}{ }^{-1}\right)$. Aunque este glucósido sea removido en su mayor parte durante el procesamiento de las raíces, debido al uso de condiciones inadecuadas de proceso, sobre todo de variedades bravas; puede estar presente en la forma intacta o hidrolizada (cianidrina y $\mathrm{HCN}$ ), en los productos derivados de la planta en concentraciones inapropiadas para el consumo, corriendo el riesgo la salud del consumidor.

- En el Brasil, la yuca es materia prima de numerosos derivados: harinas de mesa, fécula, polvillos, beiju, tapioca, carimã o masa puba, hojas o harinas de hojas y tucupi. Una análisis del flujograma general de proceso de tucupi, harina, polvillos y fécula, permite concluir que etapas como fermentación y calentamiento a ebullición (en el caso del tucupi), desmembramiento, prensado y asado (en el caso de harinas), triturado, lavado, fermentación y secado (en el caso de polvillo y fécula), son efectivas en la reducción o eliminación total del potencial toxicológico de variedades con altas concentraciones de cianógenos.

\section{BIBLIOGRAFÍA}

Agbor-Egbe, T. y Lape, I. 2006. The effects of processing techniques in reducing cyanogen levels during the production of some Cameroonian cassava foods. Journal of Food Composition and Analysis 19(4):354-363

ABIA (ASSOCIAÇÃO BRASILEIRA DAS INDÚSTRIAS DE ALIMENTOS). 2000. Compêndio da legislação de alimentos. Ministério da Salud. São Paulo, p1

Balagopalan, C. 2002. Cassava utilization in food, feed and industry. Cassava: Biology, Production and Utilization, CAB International, Kerala 15:301-318
Barros, G. 2004. Melhoria da competitividade da cadeia agroindustrial de yuca no Estado de São Paulo. São Paulo: SEBRAE, Piracicaba, ESALQ-CEPEA, 347p

Bokanga, M. 1994. Processing of cassava leaves of human consumption. Acta Horticulturae 375:203-207

BRASIL. 1978. Ministério da Salud. Resolução n. 12/78 da Comissão Nacional de Normas e Padrões para Alimentos. Aprova as normas técnicas especiais do Estado de São Paulo, revistas pelo CNNPA, relativas a alimentos e bebidas. 
Diário Oficial da República Federativa do Brasil, Brasilia, URL: http://e-legis.bvs.br/leisref/public/ show Act.php?id=16216 \#'> . [Accedido: 30 - 05 - 2008]

Cabral, L.; Souza, A.; Ando, A.; Veasey, A. y Cardoso, R. 2002. Isoenzymatic variability of cassava accessions from different regions in Brazil. Scientia Agrícola 59(3):521-527

Cagnon, R.; Cereda, P. y Pantarotto, S. 2002. Cultura de tuberosas amiláceas latino-americanas. Serie: Culturas de Tuberosas Amiláceas latino Americanas, Cd-rom, Fundação Cargill, 2(2):83-99

Cardoso, L. y Souza, S. 2002. Importância, potencialidades e perspectivas do cultivo da yuca na américa Latina. Serie: Culturas de Tuberosas Amiláceas latino Americanas, Cdrom, Fundação Cargill, 2(2):29-47

Cardoso, L. 2003. Competitividade e inovação tecnológica na cadeia agroindustrial de fécula de yuca no Brasil. Tese Doutorado em Ciências-Economia Aplicada, Escola Superior de Agricultura "Luiz de Queiroz", Piracicaba-SP, 188pp

Carvalho. C. 1992. Disponibilidade de resíduos agro-industriais e do beneficiamento de productos agrícolas. Informações Econômicas, EMBRAPA, Sao Carlos, p7-27

Cereda, P. 1987. Tecnologia e qualidade do polvillo azedo. Informe Agropecuário, Belo Horizonte 13(145):63-68

Cereda, P. 2003a. Procesamiento da Yuca como mecanismo de detoxificação.
Série: Cultura de tuberosas amiláceas latino-americanas, Cdrom, Fundação Cargill, 3(3):47-80

Cereda, P. 2003b. Cultivo de yuca. CPT, Viçosa-MG, $134 p$

Cereda, P. 1994b. Resíduos da industrialização de yuca no Brasil. Paulicieia, São Paulo, 174p

Cereda, P. y Lima, L. 1981. Aspectos sobre a Fermentação de Yuca: II - Controle das fermentações realizadas em laboratório. Boletim da Sociedade Brasileira de Ciência e Tecnologia de Alimentos 15 (2):107-122

Chisté, C.; Cohen, O. y Oliveira, S. 2005. Determinação de cianeto durante as etapas de procesamiento da harina de yuca do grupo seca. III Seminário de Iniciação Cientifica da UFRA e IX da Embrapa Amazônia Oriental. URL: http://www.artigocientifico.com.br/ acervo/5/53/tpl_1589.html.gz. [Accedido: 27 - 05 - 2008]

Chisté, C.; Cohen, O. y Oliveira, S. 2007. Estudo das propriedades físico-químicas do tucupi. Ciência e Tecnologia de Alimentos 27(3):787-792

CIAT. 2007. Corte transversal da raíz de yuca. URL: http://www.ciat.CGiar. org/. [Accedido 29 -05 - 2008]

Cohen, O.; Oliveira, S. y Chisté, C. 2007. Quantificação de teores de compostos cianogênicos totais em productos elaborados com raíces de yuca. Belém (PA): Embrapa Amazônia Oriental, p11-21

Corrêa, D. 2000. Harina de folhas de yuca (Manihot esculenta cv Baiana) - 
Efeito de procesamientos sobre alguns nutrientes e antinutrientes. Tesis Ph. D., Universidade Federal de Lavras, Lavras

Dallaqua, M. y Coral, J. 2002. MorfoAnatomia. Cultura de tuberosas amiláceas Latino Americanas, CD ROM, Fundação Cargill, 2(3):48-65

Damardjati, S.; Widowati, S. y Rachim, A. 1993. Cassava flour production and consumers acceptance at village level in Indonesia. Indonesian Agricultural Research and Development Journal 15:16-25

Demiate, M.; Barana, C.; Cereda, P. y Wosiacki, G. 1999. Organic acid profile of commercial sour cassava starch. Ciência e Tecnologia de Alimentos 19(1):131-135

Dias, C.; Longhi, A. y Lorenzi, O. 1997. Yuca: Manihot esculenta Crantz. Manual técnico das culturas. CATI, Campinas, 8(1):369-98

Djazuli, M. y Bradbury, H. 1999.Cyanogen content of cassava roots and flour in Indonesia. Food Chemistry 65(4):523-525

Du, L.; Bokanga, M.; Moller, L. y Halkier, B. 1995. The biosynthesis of cyanogenic glucosides in roots of cassava. Phytochemistry 39(2):323-326

Dufour, L. 1988. Cyanide content of cassava (Manihot esculenta, Euphorbiaceae) cultivars used by Tukanoan Indians in Northwest Amazonia. Economic Botany 42(2):255-266

EMBRAPA. Sistemas de produção de yuca. URL: http://sistemasdeproducao. c n p tia.embrapa.br\#yuca> [Accedido: 04 - 10 - 2006]

FAO/WHO. 1991. Food Standards Programme. Codex Alimentarius Commission XII Supplement 4, Joint FAO/WHO, Rome, FAO, 1:18-27

Ferreira, R. 1997. Efeito da adubação orgânica e densidade populacional na cultura da yuca em solo de tabuleiro. Revista Brasileira de Yuca 16(1):7-14

Fukuda, C. y Otsubo, A. 2006. Cultivo da yuca na região centro sul do Brasil. URL: http://sistemasdeproducao. cnptia.embrapa.br. [Accedido: 30 09 - 2006]

IBGE (Instituto Brasileiro de Geografia e Estatística). 2006. Produção de yuca em 2004. URL: http://www.ibge. gov.br [Accedido: 29 - 05 - 2008]

Ikediob, C.; Onyia, G. y Eluwah, C. 1980. A rapid inexpensive enzymatic assay for total cyanide in cassava product. Agricultural and Biological Chemistry 44:2803-2809

Iwuoha, I.; Banigo, I. y Okwelum, C. 1997. Cyanide content and sensory quality of (Manihot esculenta Crantz) root tuber affected by processing Cassava flour. Food Chemistry 58(4):285-288

Jones, M.; Trim, S.; Bainbridge, A. y French, L. 1994. Influence of selected process variables on the elimination of cyanide from cassava. Journal of the Science of Food and Agriculture 66(4):535-542

Leonel, M. y Cereda, P. 2000. Extração da fécula retida no resíduo fibroso do 
processo de produção de fécula de yuca. Ciência e Tecnologia de Alimentos 20(1):121-127

Lorenzi, O. 2003. Yuca. Boletim Técnico, CATI, Campinas, 245:116

Lorenzi, O.; Ramos, B.; Monteiro, A.; Valle, L. y Godoy, G. 1993. Teor de ácido cianídrico em variedades de yuca cultivadas em quintais do Estado de São Paulo. Bragantia 52(1):1-5

Mattos, P. y Bezerra, S. O Cultivo da Yuca para o Estado do Amapá. URL: http:/ /www.embrapa.br [Accedido: 26 09 - 2006]

Mattos, P.; Gomes, C.; Farias, N. y Fukuda, C. 2002. Cultivo da yuca nas regiões Norte e Nordeste do Brasil. Cultura de tuberosas amiláceas Latino Americanas, Fundação Cargill, 2 (14):274-301

Mcmahon, C.; White, B. y Sayre, T. 1995. Cyanogenesis in cassava (Manihot esculenta Crantz). Journal of Experimental Botany 46(7):731-741

Mkpong, E.; Yan, H.; Chism, G. y Sayre, T. 1990. Purification, characterisation and localisation of linamarase in cassava. Plant Physiology 93(1):176-181

Ngudi, D.; Kuo, H. y Lambein, F. 2003. Cassava cyanogens and free amino acids in raw and cooked leaves. Food and Chemical Toxicology 41(8):1193-1197

Normanha, S. 1988. O mau cozimento dos aipins: uma hipótese. Agronômico $40(1): 13-14$
Oliveira, N. 1986. Sombra considerações sobre os princípios tóxicos da yuca. UEPAR, Brasília, $14 p$

Pereira, S.; Lorenzi, O. y Valle, L. 1985. Avaliação do tempo de cozimento e padrão de massa cozida em yuca de mesa. Revista Brasileira de Yuca $4(1): 27-32$

Souza, S. y Fialho, F. Cultivo da Yuca para a Região do Cerrado: Colheita e Póscolheita. URL: http:// sistemasdeproducao.cnptia.embrapa.br [Accedido: 30 - 09 - 2006]

Taiz, L. y Zeiger, E. 2004. Fisiologia Vegetal. Artmed, Porto Alegre, 719p

Tylleskar, T.; Banea, M.; Bikangi, N.; Cooke, R.; Poulter, N. y Rosling, H. 1992. Cassava cyanogens and konzo, an upper motor neuron disease found in Africa. The Lancet 339(87):208-211

Valle, L.; Carvalho, L.; Ramos, B.; Mühlen, S. y Villela, V. 2004. Conteúdo cianogênico em progênies de yuca originadas do cruzamento de variedades mansas e bravas. Bragantia, 63(2):221-226

Wajant, H. y Pfizenmaier, K. 1996. Identification of potential activesite residues in the hydroxynitrile lyase from Manihot esculenta by site-directed mutagenesis. The Journal of Biology Chemistry 271(42):25830-25834

Yeoh, H. 1989. Kinetic properties of betaglucosidase from cassava. Phytochemistry 28(3):721-724 